\title{
The chemical phụsics of the photostability of life
}

\author{
Andrzej L. Sobolewski ${ }^{1}$ and Wolfgang Domcke ${ }^{2}$ \\ 'Institute of Physics, Polish Academy of Sciences, PL-02668 Warsaw.Poland•Email: sobola@ifpan.edu.pl \\ 2 Department of Chemistry, Technical University of Munich, D-85747 Garching • Germany
}

\begin{abstract}
Tife appears as incredibly complex when it is investigated with atomic resolution. However, the vast majority of biological matter consists of rather few building blocks, which are comparatively simple organic molecules. The building blocks of life are, first of all, the four nuclei acid bases adenine, cytosine, guanine and thymine (see Scheme I), which encode the genetic information of all living creatures in DNA (deoxyribonucleic acid). The exceedingly multifarious world of proteins is built from only 20 amino acids (a few of them are shown in Scheme II). Another widespread molecular motif are carbohydrates (e.g., sugars). Sugar molecules are part of the backbone of DNA and of structure-forming biopolymers such as cellulose.

Organic molecules are not stable under persistent irradiation with ultraviolet (UV) light. UV photons can break covalent bonds and thus can induce a great variety of chemical transformations (isomerizations or fragmentations). In view of this, it is amazing that life can thrive under full exposition to sunlight. Moreover, biogenesis took place long before the formation of the stratospheric ozone layer (which today filters out the most dangerous UV components of sunlight) and thus under conditions of extremely intense short-wavelength UV radiation. As pointed out by Sagan, this must have resulted in an extreme selection pressure for UV protection [1]. These considerations suggest that photostability may have been the decisive selection criterion which has determined the molecular architecture of life at the beginning of the biological evolution.

In this article, we discuss recent theoretical and experimental results which support the hypothesis that the fundamental building blocks as well as the supramolecular structures of life are optimized with respect to photostability.
\end{abstract}

\section{Photostability}

The concept of photostability and the physical mechanisms providing photostability can best be explained by referring to so-called photostabilizers [2], which are in widespread technical use for the protection of organic polymers. Photostabilizers are organic compounds (usually intramolecularly hydrogen-bonded aromatic molecules) which absorb UV photons with a large cross section and convert the photon energy into heat, without undergoing destructive photochemical reactions. The best photostabilizers can neutralize nearly a million of photons before being destroyed by a photochemical reaction. The essential mechanism is ultrafast radiationless decay of the photoexcited singlet state to the electronic ground state. This process is called internal conversion. It

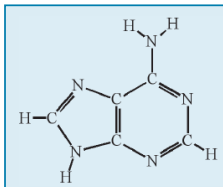

adenine

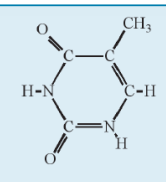

thymine

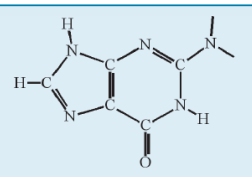

guanine

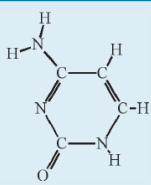

cytosine
$\Delta$ Scheme I

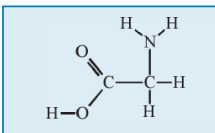

glycine

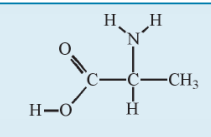

$\nabla$ Scheme II

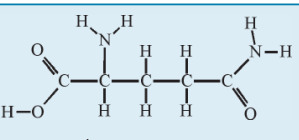

alanine

glutamine converts the potentially dangerous energy of the UV photon into vibrational energy (heat) which is subsequently dissipated into the environment. As we shall discuss below, the DNA bases and especially the supramolecular structures of DNA and proteins avail themselves of very efficient excited-state deactivation mechanisms which are similar to those of commercial photostabilizers.

\section{Potential-energy surfaces,} conical intersections and radiationless decay

To explain the physical mechanisms of photostability, it is necessary to introduce two fundamental theoretical concepts. The first concept is the Born-Oppenheimer (BO) potential-energy surface. As is well known, the $\mathrm{BO}$ approximation is based on the large mass difference between electrons and nuclei, resulting in a separation of time scales. It is usually a good approximation to assume that the fast electrons follow instantaneously the much slower motion of the nuclei. In this $\mathrm{BO}$ adiabatic approximation, the nuclear motion is thus tied to a particular eigenvalue of the electronic Schrödinger equation, the so-called $\mathrm{BO}$ potential-energy surface. The second concept is the conical intersection of $\mathrm{BO}$ potential-energy surfaces, dating back to a fundamental paper by von Neumann und Wigner [3]. Von Neumann and Wigner realized that in a polyatomic molecule, other than in diatomic molecules, $\mathrm{BO}$ energy surfaces generically are allowed to have exact crossings. These crossings are called conical intersections, since the energy surfaces have the shape of a double cone in a suitable two-dimensional subspace of the nuclear coordinates. At these conical intersections, the $\mathrm{BO}$ adiabatic approximation is strictly invalid, since the nonadiabatic couplings, which usually are

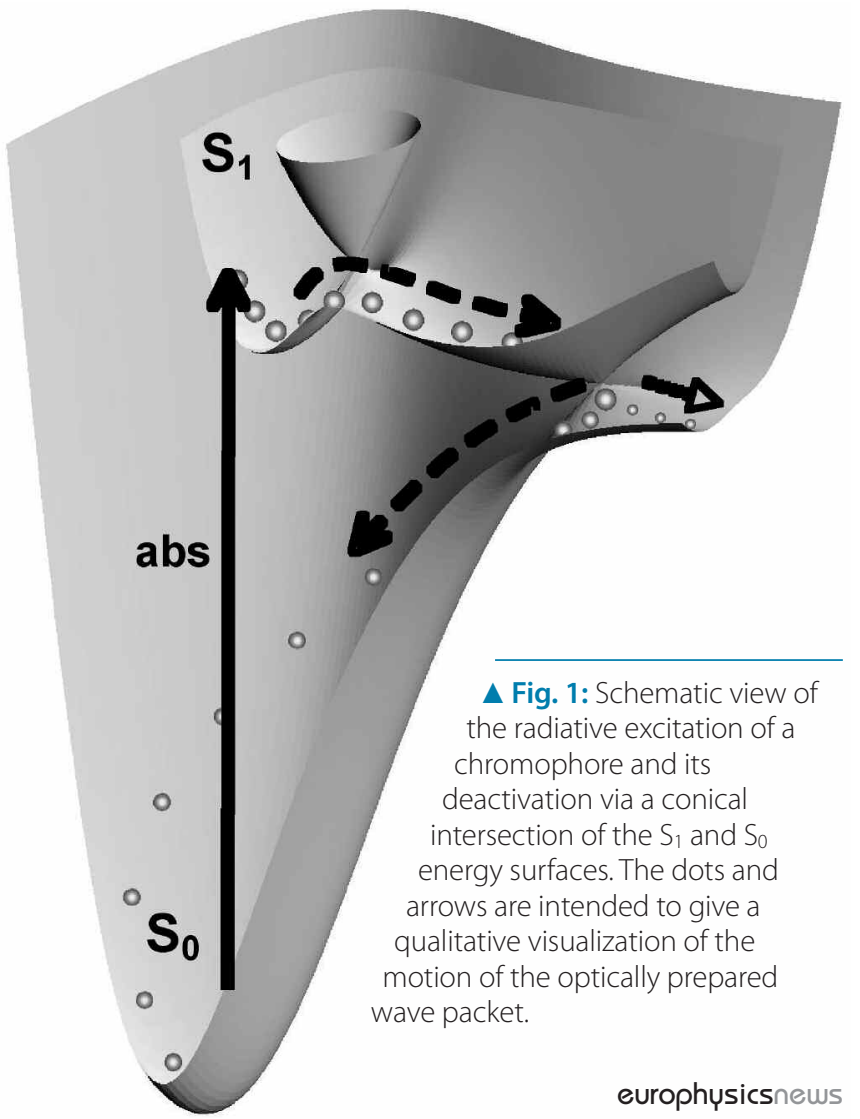


neglected, become infinite. The molecule thus can switch extremely efficiently from the energy surface of the excited state to the energy surface of the electronic ground state at such conical intersections. Extensive research in computational chemistry during the last decade has revealed that (i) conical intersections are indeed ubiquitous in polyatomic molecules $[4,5]$ and (ii) internal conversion at conical intersections is essentially instantaneous, that is, it occurs within a fraction of the relevant vibrational periods $[6,7]$. Conical intersections thus are the key mechanistic elements for photostability, provided they are accessible in a barrierless manner on the excitedstate potential-energy surface. A schematic view of the radiative excitation process and the rapid radiationless deactivation process via a conical intersection is shown in Fig. 1. While the excitation process is "vertical", that is, occurs at the ground-state equilibrium geometry of the molecule, the deactivation process involves a photochemical reaction which is aborted at the conical intersection.

It is clear from this brief outline that a reliable first-principles characterization of the electronic potential-energy surfaces is essential for the theoretical understanding of photostability. We cannot cover here the rather involved technical aspects of these calculations. Detailed information about the electronic-structure methods can be found in the quoted literature.

\section{Photostability of isolated DNA bases}

Photostability of the molecular encoding of genetic information clearly is of utmost importance for the existence of life. Although the DNA bases absorb strongly in the $200-300 \mathrm{~nm}$ range, the quantum yields of their photoproducts are very low. The kinetics of the radiationless decay of individual DNA bases has been investigated in the gas phase as well as in solution, see [8] for a recent review. The measured lifetimes indeed are very short, of the order of a few hundred femtoseconds in solution [8].

It has recently been shown by several research groups that the methods of ab initio quantum chemistry can contribute significantly to the identification of the excited-state deactivation mechanisms of isolated DNA bases (see [9] and references therein). Two types of photochemical reaction paths, which lead to low-lying conical intersections of excited-state and ground-state energy surfaces, have been identified: (i) the torsion of certain C-N bonds of the sixmembered rings, and (ii) the abstraction of hydrogen atoms from azine or amino groups. Fig. 2 shows, as an example, the energy profiles of the electronic ground state $\left(\mathrm{S}_{0}\right)$ and the three lowest excited states of adenine as a function of the reaction coordinate (primarily torsion of the $\mathrm{C}_{2} \mathrm{~N}_{3}$ bond of the six-membered ring of adenine) leading to the lowest-energy conical intersection.

The curve crossings, which are accentuated by colored circles in Fig. 2, become conical intersections when the remaining nuclear degrees of freedom are taken into account. It is seen that the stabilization of the second excited singlet state of $\pi \pi^{*}$ character $\left(\pi / \pi^{*}\right.$ are occupied/unoccupied molecular orbitals with a node in the molecular plane) leads to a conical intersection of this state with the electronic ground state. This intersection is lower in energy than the

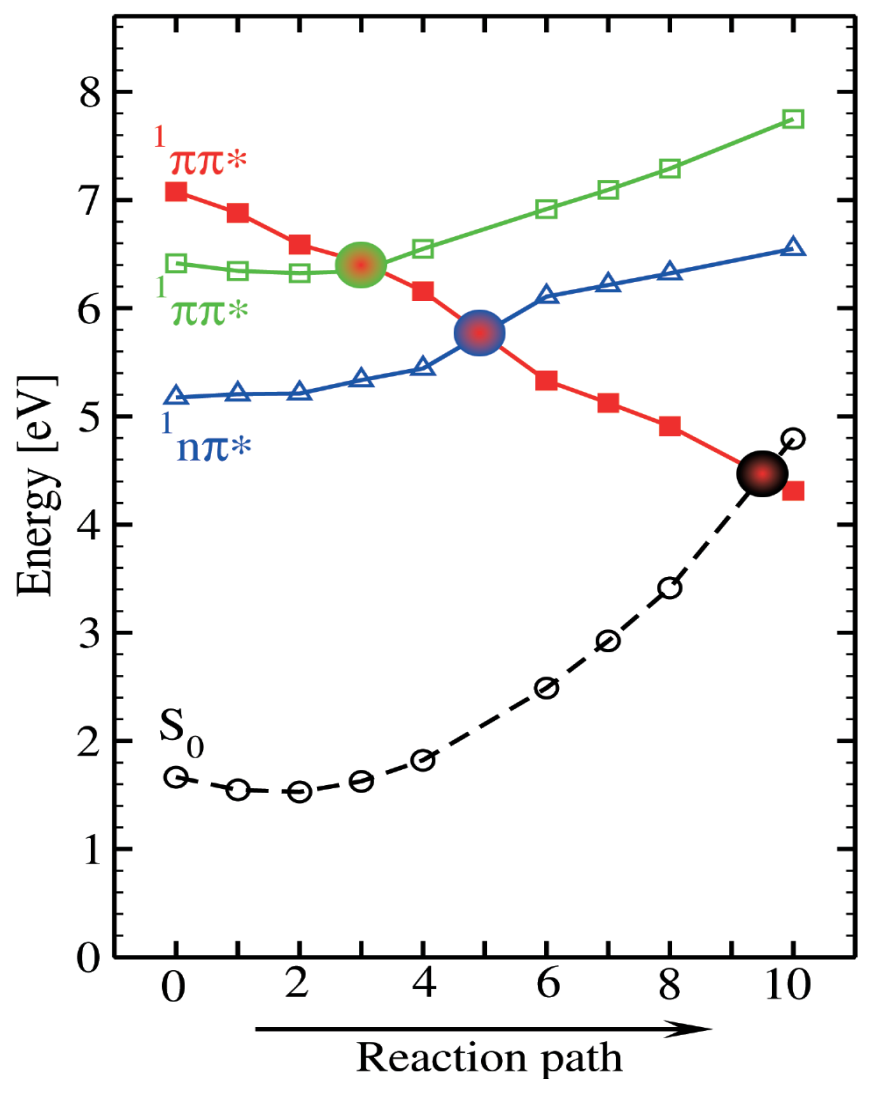

$\Delta$ Fig. 2: Potential-energy profiles of the ground state $\left(S_{0}\right)$ and the lowest excited singlet states ('n $\pi^{*}{ }^{*}{ }^{1} \pi \pi^{*}$ ) of adenine along the reaction path leading from the ground-state equilibrium geometry (left) to the $S_{1}-S_{0}$ conical intersection (right).

minimum of the lowest excited singlet state ( ${ }^{1} \mathrm{n} \pi^{*}$, where $\mathrm{n}$ denotes a nonbonding molecular orbital) of adenine. It can be reached in a nearly barrierless manner from the first ${ }^{1} \pi \pi^{*}$ state, which is the lowest UV absorbing state of adenine. Similar results have been obtained for cytosine, guanine and thymine. Taken together, these computational results confirm the existence of very efficient channels of radiationless deactivation in the DNA bases. The DNA bases thus appear to be optimized with respect to photostability. This property can explain the selection of just these four species at the beginning of the biological evolution. They may have played the role of integrated sunscreens in the earliest biopolymers (presumably RNA).

\section{Photostability of DNA base pairs}

It is a most remarkable feature of life that only two base pairs (adenine-thymine (AT) and guanine-cytosine (GC)) encode all the genetic information. Recently, is has become possible to obtain the electronic and vibrational spectra of these hydrogen-bonded base (a)

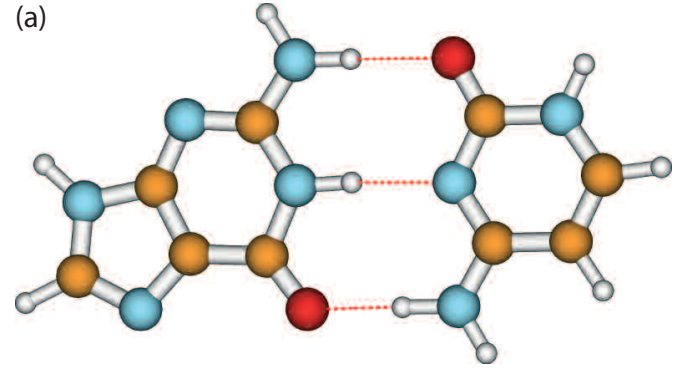

(b)

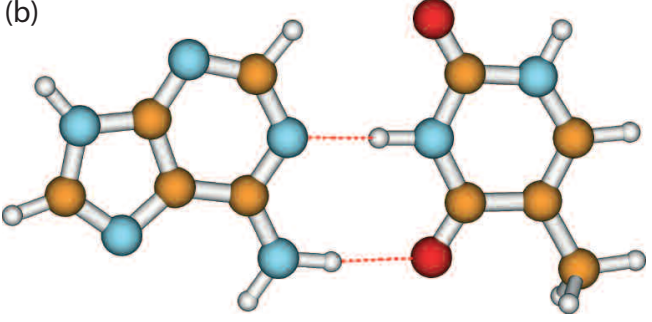

4 Fig. 3: Equilibrium structures of the guanine-cytosine (a) and adenine-thymine (b) Watson-Crick base pairs. Colour code: yellow: carbon; blue: nitrogen; red: oxygen; grey: hydrogen. 


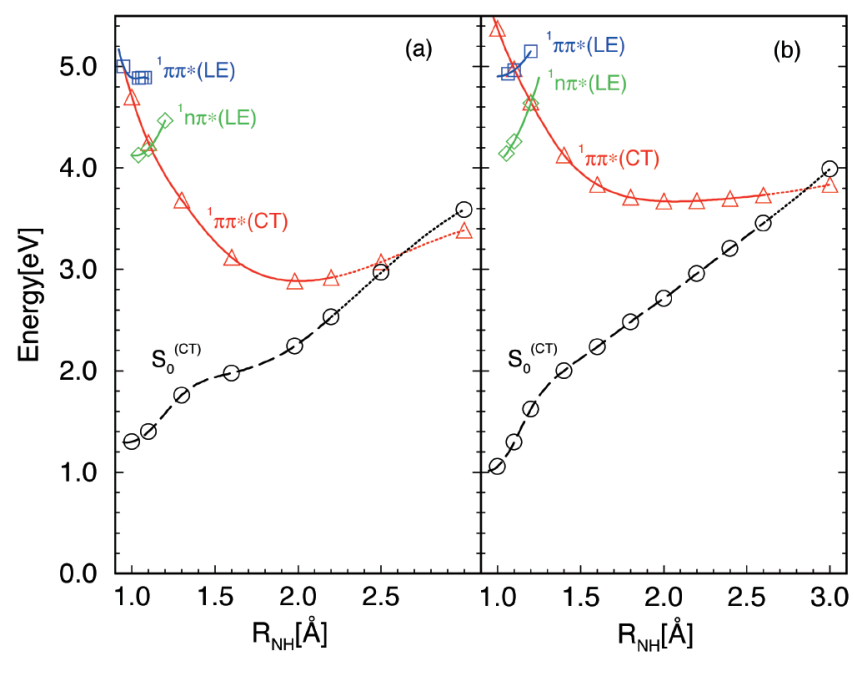

$\Delta$ Fig. 4: Potential-energies of the ground state $\left(\mathrm{S}_{0}\right)$, the lowest locally-excited states ('n$\left.\pi^{*}(\mathrm{LE}),{ }^{1} \pi \pi^{*}(\mathrm{LE})\right)$ and the lowest chargetransfer state $\left({ }^{1} \pi \pi^{*}(C T)\right)$ as functions of the proton-transfer coordinate in the GC (a) and AT (b) Watson-Crick base pairs. The excited-state energies have been calculated at the minimum-energy geometries of the respective states, while this designated as $\mathrm{S}_{0}{ }^{(\mathrm{CT})}$ has been determined at the minimum-energy geometries of the charge-transfer state

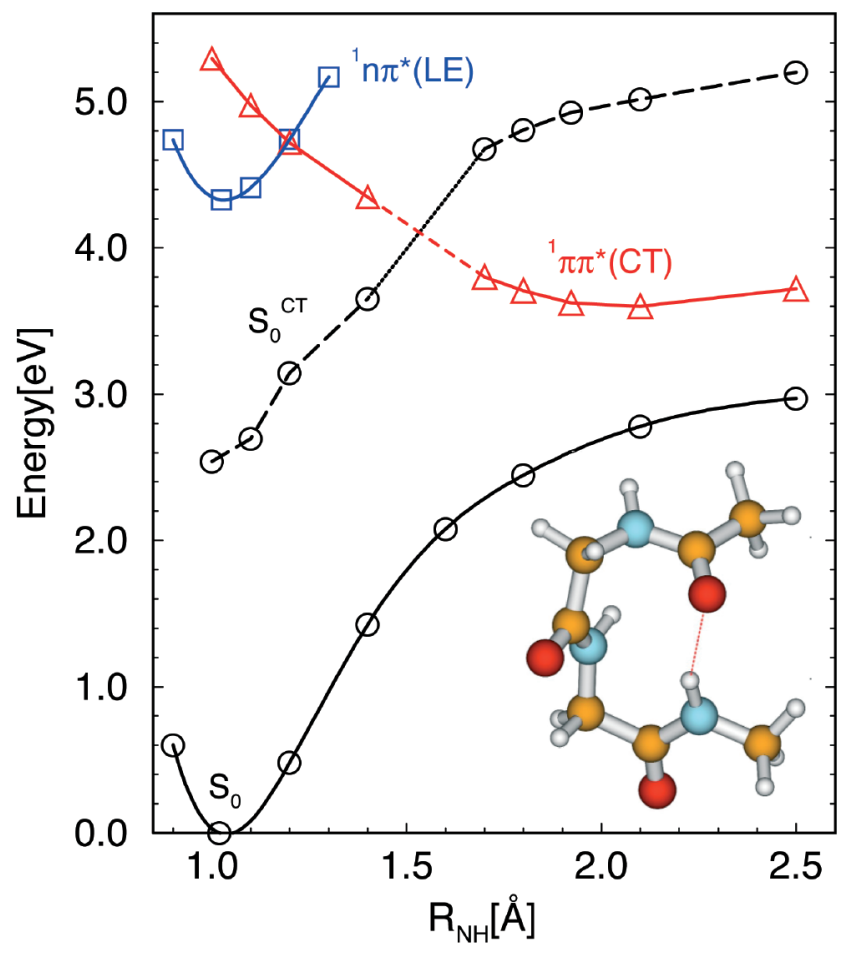

$\Delta$ Fig. 5: Potential-energies of the locally-excited state (blue), the charge-transfer state (red) and the electronic ground state (black) as functions of the proton-transfer coordinate in the glycine trimer. The ${ }^{\prime} n \pi^{*}(\mathrm{LE})$ and ${ }^{\prime} \pi \pi^{*}(\mathrm{CT})$ energies have been determined at the minimum-energy geometries of the respective states. The groundstate energies designated as $S_{0}$ have been calculated at the minimum-energy geometries of the $\mathrm{S}_{0}$ state, while those designated as $\mathrm{S}_{0}{ }^{\mathrm{CT}}$ have been determined at the minimum-energy geometries of the charge-transfer state. pairs in isolation by laser evaporation and subsequent cooling in a supersonic jet. The isolated AT and GC base pairs can exist in a variety of structures (so-called conformers) which differ in the hydrogen-bonding geometry. With sophisticated double-resonance laser techniques and first-principles calculations of the vibrational spectra, it has been possible to assign the observed spectra to specific conformers [10]. The so-called Watson-Crick conformers of AT and GC, that are the only ones relevant for biology, are displayed in Fig. 3. It is seen that GC (AT) are connected by three (two) hydrogen bonds (dotted lines). For the GC base pair, the spectra of three conformers have been detected $[10,11]$. Remarkably, the resonantly enhanced multi-photon ionization spectrum of the Watson-Crick conformer of GC is extremely weak and broad, in sharp contrast to the strong and sharp signals of the two other conformers [11]. An excited-state lifetime of a few femtoseconds has been estimated from the spectra of the Watson-Crick conformer of GC. The UV spectrum of the Watson-Crick conformer of AT has not yet been observed.

Calculations of the potential-energy functions of GC and AT have led to the discovery of a new mechanism of excited-state deactivation which is specific for hydrogen-bonded aromatic systems [12]. The calculations have revealed that electron-driven proton-transfer processes between the bases play an important role in the photophysics of these systems. The key player in this mechanism is a "dark" charge-transfer (CT) state which connects the optically accessible locally-excited state with the electronic ground state via two conical intersections along the proton-transfer coordinate. The exceptionally short lifetime of the UV-absorbing states of the Watson-Crick conformer of the GC base pair is explained by a barrierless access to the reactive charge-transfer state, see Fig. 4a, which provides the mechanism for fast return of the photoexcited system to the electronic ground state. In the two other conformers of GC, the photochemically reactive charge-transfer state is located higher in energy, resulting in a barrier along the proton-transfer reaction path and thus a considerably longer excited-state lifetime [12]. The same qualitative features are predicted by the calculations for the AT base pair: only in the Watson-Crick conformer the locally-excited state is crossed near its minimum by the reactive charge-transfer state, see Fig. 4b [12]. These results suggest that the biologically relevant Watson-Crick structures of GC and AT are distinguished by uniquely efficient excited-state deactivation mechanisms which maximize their photostability.

Additional evidence for enhanced excited-state deactivation via the electron-driven proton-transfer mechanism has also been found for a biomimetic model of the Watson-Crick base pairs, the 2 -aminopyridine dimer. Femtosecond time-resolved pump-probe measurements have revealed that the excited-state lifetime is shortened by a factor of twenty in the hydrogen-bonded dimer [13]. Calculations have predicted the existence of a reactive chargetransfer state in the dimer, which connects the locally-excited state with the electronic ground state via two conical intersections [13].

\section{Photostability of proteins}

The so-called secondary structure of folded native proteins is determined to a large extent by hydrogen bonds between $\mathrm{NH}$ and $\mathrm{CO}$ groups of the backbone. Well-known structural motifs in proteins are $\alpha$-helices and $\beta$-sheets. At present, ab initio electronic-structure calculations of excited electronic states are possible only for relatively small oligomers of amino acids, which are called peptides.

The molecular structure of a small peptide, the glycine trimer, is shown as inset in Fig. 5. The dangling bonds of the backbone have been capped by methyl groups. This structure is a model of a so-called $\beta$-turn of the peptide backbone which is stabilized by a $\mathrm{C}=\mathrm{O}$... H-N hydrogen bond (dotted line). 
An analysis of the excited electronic states of this model peptide reveals that these can be classified, in a qualitative sense, as locally-excited and charge-transfer states [14]. The lowest locallyexcited state involves excitation of an electron from the highest occupied molecular orbital to the lowest unoccupied molecular orbital, which are both localized on the lower branch of the backbone. The lowest charge-transfer state, on the other hand, involves excitation from an occupied molecular orbital on the lower branch to an unoccupied orbital on the upper brach of the backbone. Similar to what has been found for the DNA base pairs, the proton of the $\mathrm{NH}$ group wants to follow the electron, leading to a pronounced stabilization of the energy of the charge-transfer state by proton transfer. As shown in Fig. 5, the energy surface of the charge-transfer state (red) crosses the energy surfaces of both the locally-excited state (blue) as well as the ground state (black). Again the energy surface of the locally-excited state, which is generated by the absorption of a UV photon, is connected via two conical intersections with the energy surface of the ground state. These conical intersections provide the mechanism for the ultrafast deactivation of the excited state.

\section{Conclusions}

Hydrogen bonds are ubiquitous in biological matter. Their universal role in structure formation, molecular recognition and catalysis is well known [15]. Life on earth thus depends essentially on the functionality of hydrogen bonds.

Herein, we have provided evidence that the photoinduced excited-state chemistry of hydrogen bonds may be as essential for the existence of life as the ground-state chemistry. As has been discussed above for the examples of DNA base pairs and model peptides, the photochemistry of hydrogen bonds provides a mechanism for the deactivation of the potentially reactive excited states which is of unrivalled efficiency.

The overall radiationless deactivation process may be visualized as an electron-driven proton-transfer mechanism as schematically indicated in Fig. 6. Once the charge-transfer state is populated, the proton moves from the donor atom towards the acceptor atom, following the electron; after the back-transfer of the electron at the conical intersection of the charge-transfer state with the $S_{0}$ state, the proton is driven back to the original location. This way, the energy of the UV photon is converted into vibrational energy of the hydrogen bond, which subsequently is dissipated to other intramolecular as well as intermolecular vibrational degrees of freedom. The unusually large electronic energy gradients and the small mass of the proton ensure the exceptionally fast rate of this process. The photoexcited biomolecule thus returns to the closed-shell electronic ground state before deleterious photochemical reactions can take place..

\section{About the Authors}

Andrzej L. Sobolewski studied physics at the University of Warsaw and received his $\mathrm{PhD}$ in the Institute of Physics of the Polish Academy of Sciences in 1981. Since 1989 he has been Professor of

- Fig. 6: Schematic view of ultrafast excited-state deactivation via the electron-driven proton-transfer process: the locally excited (LE) electronic state is deactivated via two sequential electron-transfer (ET) processes at conical intersections of the locally-excited state (blue) and the electronic ground state (black) with a charge-transfer state (red). After each electron transfer, the proton follows the electron, thus accepting the energy deposited by the UV photon in the molecular system.
Physics at this Institute. His research interests are concerned with different aspects of the theory of molecular photophysics.

Wolfgang Domcke studied physics and received his $\mathrm{PhD}$ at the Technical University of Munich in 1975. He has been Professor of Theoretical Chemistry at the Universities of Heidelberg, Düsseldorf and, since 1999, at the Technical University of Munich. His research interests comprise molecular electronic-structure theory as well as theoretical aspects of chemical reaction dynamics and molecular spectroscopy.

\section{References}

[1] C. Sagan, J. theor. Biol. 39, 195 (1973).

[2] J.-E. A. Otterstedt, J. Chem. Phys. 58, 5716 (1973).

[3] J. von Neumann and E. Wigner, Physik. Z. 30, 467 (1929).

[4] D. R. Yarkony, Rev. Mod. Phys. 68, 985 (1996).

[5] M. A. Robb, F. Bernardi and M. Olivucci, Pure and Appl. Chem. 67, 783 (1995).

[6] W. Domcke and G. Stock, Adv. Chem. Phys. 100, 1 (1997).

[7] W. Domcke, D.R. Yarkony and H. Köppel (Eds.), Conical Intersections: Electronic Structure, Dynamics and Spectroscopy, World Scientific, Singapore, 2004.

[8] C.E. Crespo-Hernandez, B. Cohen, P.M. Hare and B. Kohler, Chem. Rev. 104, 1977 (2004).

[9] S. Perun, A. L. Sobolewski and W. Domcke, J. Am. Chem. Soc. 127, 6257 (2005); see also A.L. Sobolewski, C. Woywod and W. Domcke, J. Chem. Phys. 98, 5627 (1993).

[10] E. Nir, C. Janzen, P. Imhof, K. Kleinermanns and M.S. de Vries, Phys. Chem. Chem. Phys. 4, 732 (2002); C.Plützer, I. Hünig, K. Kleinermanns, E. Nir and M.S. de Vries, ChemPhysChem 4, 838 (2003).

[11] A. Abo-Rizig, L. Grace, E. Nir, M. Kabelac, P. Hobza and M.S. de Vries, Proc. Natl. Acad. Sci. USA 102, 20 (2005).

[12] A.L. Sobolewski and W. Domcke, Proc. Natl. Acad. Sci. USA 102, 17903 (2005); S. Perun, A.L. Sobolewski and W. Domcke, J. Phys. Chem. A, in press.

[13] T. Schultz, E. Somoylova, W. Radloff, I.V. Hertel, A.L. Sobolewski and W. Domcke, Science 306, 1765 (2004).

[14] A.L. Sobolewski and W. Domcke, ChemPhysChem. 7, 561 (2006).

[15] P. Schuster, G. Zundel and C. Sandorfy, The Hydrogen Bond - Recent Developments in Theory and Experiments. II. Structure and Spectroscopy, North Holland, Amsterdam, 1976.

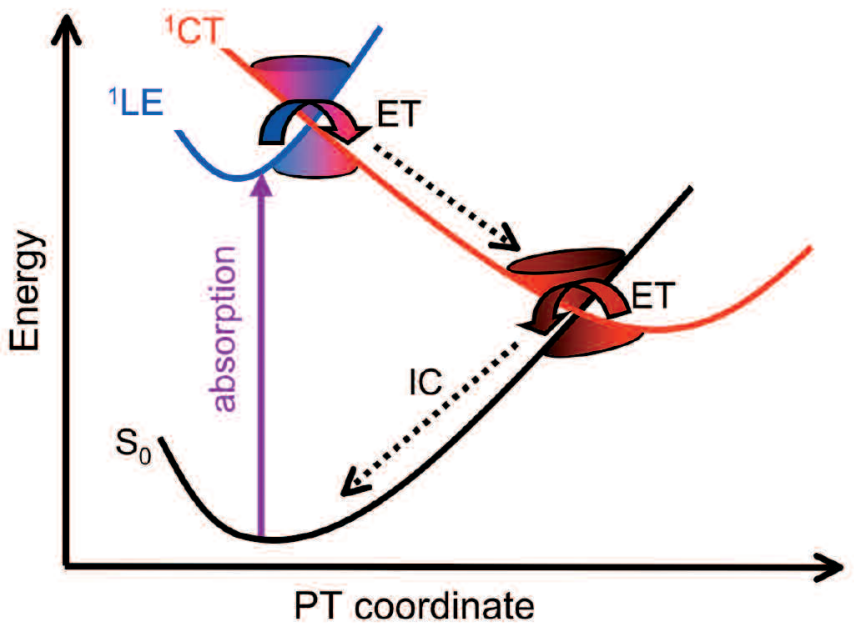

